\title{
The Utility of Extracorporeal Membrane Oxygenation in Patients With Hematologic Malignancies: A Literature Review
}

\author{
Praveena Tathineni ${ }^{1}$, Mauna Pandya ${ }^{2}$, Bassem Chaar $^{3}$ \\ 1. Internal Medicine, University of Illinois at Chicago/Advocate Christ Medical Center, Chicago, USA 2. Hematology \\ and Oncology, Advocate Christ Medical Center, Chicago, USA 3. Hematology and Oncology, University of Illinois at \\ Chicago/Advocate Christ Medical Center, Chicago, USA
}

Corresponding author: Praveena Tathineni, praveena.tathineni@gmail.com

\begin{abstract}
Extracorporeal membrane oxygenation (ECMO) is used to provide respiratory and/or circulatory support for critically ill patients. In people suffering from hematologic malignancies (HMs), acute respiratory failure often necessitates intensive care. Whereas initial studies reported that these patients generally have poor outcomes, studies conducted within the last 10 years have shown that ECMO is quite beneficial for patients with HMs. This review showcases data from 2010 to 2019 demonstrating the utility of ECMO in cancer patients. Retrospective studies revealed long-term disease-free survival, particularly when ECMO served as a bridge through chemotherapy. Case reports suggested strong evidence of mortality benefit from ECMO, especially in patients with aggressive lymphomas. However, a systematic approach is needed to better quantify and validate these findings. Studies with larger sample size and prospective cohorts are needed to help create well-defined guidelines for physicians approaching the treatment of cancer patients on ECMO.
\end{abstract}

Categories: Internal Medicine, Oncology, Hematology

Keywords: oncology, chemotherapy, lymphoma, ecmo, malignant hematology

\section{Introduction And Background}

Extracorporeal membrane oxygenation (ECMO) is a device used to drain blood from the venous system, oxygenate it with an artificial filter, and return it to the venous or arterial supply - acting as a cardiopulmonary bypass [1]. During the last decade, the use of ECMO in the intensive care unit (ICU) has exponentially increased. It is generally indicated for patients suffering from acute respiratory distress syndrome (ARDS), and/or cardiogenic shock that is refractory to maximal medical management, including inotropic support and/or placement of an intra-aortic balloon pump [2]. Historically, ECMO has been used to provide both circulatory and respiratory support. Veno-arterial (VA) ECMO is used in cardiogenic shock to maintain a patient's systemic circulation, functioning as a bridge to myocardial tissue recovery, destination therapy with left ventricular assist device placement or orthotopic heart transplant, or coronary artery bypass surgery [2]. It involves inserting a percutaneous cannula to drain blood from the inferior vena cava to the ECMO circuit and deliver newly oxygenated blood to the aorta via the femoral artery [3]. Veno-venous (VV) ECMO involves inserting a large bore cannula in the femoral vein or internal jugular vein to drain blood from the inferior vena cava and pass it through the ECMO circuit for oxygenation and removal of carbon dioxide. The filtered blood is returned to the right atrium via the superior vena cava, thus helping maintain gas exchange [3].

Tathineni et al. This is an open access article distributed under the terms of the Creative Commons Attribution License CC-BY 4.0., which permits unrestricted use, distribution, and reproduction in any medium, provided the original author and source are credited.

In patients with hematologic malignancies (HMs) such as leukemia, lymphoma, and multiple myeloma, acute respiratory failure is the most common diagnosis warranting ICU admission [4]. While recent advances in chemotherapy and hematopoietic stem cell transplant (HSCT) have benefited these patients, up to $50 \%$ of those hospitalized eventually require mechanical ventilation [5-6]. Under such circumstances, ECMO may be used for severe circulatory and/or pulmonary dysfunction [7]; it is also used in patients with tumor lysis syndrome to help stabilize them through chemotherapy [8]. The complex nature of patients with HMs increases their risk of complications from ECMO, specifically bleeding and infections [9-11]. The additional use of chemotherapy often leads to cytopenias and extended periods of marrow aplasia, making them susceptible to bacterial, viral, and fungal infections. Hence, initiating ECMO in patients with HMs has been associated with a high-risk state [9-11].

Studies conducted in the late 20th century imply that patients with HMs on ECMO have poor outcomes [1218]. More recent analyses have demonstrated long-term disease-free survival in patients with HMs on ECMO $[4,9]$. This association of ECMO with mortality benefit in patients with HMs poses the following question: how should hematologists/oncologists manage aggressive malignancies while patients are on ECMO? A comprehensive review comprising findings from the last decade on this topic has not yet been published. The purpose of this article is to gather and analyze data from 2010 to 2019 regarding the use of ECMO in patients with HMs, and its relation to mortality. We believe that the complexity of disease in this subset of patients necessitates clearer and more defined treatment protocols for patients with HMs who require 


\section{Review}

\section{Search strategy}

The search was conducted using the PubMed database and included case reports, case series, reviews, and studies. The following keywords were used: extracorporeal membrane oxygenation, malignant hematology, oncology, chemotherapy, and lymphoma. We screened available articles from 2010 to 2019 that discussed the use of ECMO in patients suffering from aggressive HMs. This was followed by a search using phrases such as "successful treatment with chemotherapy delivered under ECMO," often accompanied by the keyword 'lymphoma.' Our search was completed by analyzing pharmacological dosing for patients on ECMO who were treated with chemotherapy.

\section{Reports of patients with HMs who required ECMO for pulmonary support}

At the start of the decade, studies involving a variety of adult cancer patients were conducted to assess the effect of extracorporeal life support on clinical outcomes. One study from 2010 included 47 solid tumor patients, 21 patients with HMs, and four post-HSCT patients - all of whom required ECMO for pulmonary support. Ultimately, $61 \%$ of this group died on ECMO, 32\% survived to hospital discharge, and $7 \%$ survived on ECMO but died before discharge [16]. This supported the general sentiment at the time that the use of ECMO in cancer patients is rarely successful from a mortality perspective. Kang et al. performed a systematic retrospective review of clinical outcomes in 15 patients with HMs who were treated with ECMO after failure of optimal conventional therapy [17]. This study was conducted in South Korea; two-thirds of the patients had a primary diagnosis of acute myelogenous leukemia or acute lymphoblastic leukemia. Their outcomes were compared to 33 immunocompetent patients with documented cardiorespiratory failure that warranted ECMO support. The patients with underlying HMs were associated with significantly higher mortality rates compared to the immunocompetent patients, partially attributed to infections and hyperbilirubinemia during ECMO [17].

In 2012, Azoulay et al. performed a prospective, observational cohort study in 17 centers in France and Belgium to assess outcomes in critically ill malignant hematology patients [9]. Amongst 1,011 patients with HMs admitted to the ICU, the mortality rate was $39.3 \%$. The subsequent disease-control rate six months after discharge from the hospital was $80 \%$ [9]. Although this study did not specifically take ECMO into account, the mortality rate was significantly lower than that established in prior studies. This was a starting point for the conclusion and realization that critically ill patients with HMs may have improved survival rates [9].

In 2014, Wohlfarth et al. conducted a retrospective cohort study of 14 adult Austrian patients with HMs, all of whom received ECMO support due to acute respiratory failure [4]. They had a variety of aggressive HMs, including non-Hodgkin lymphoma, Hodgkin lymphoma, acute myeloid leukemia, and multiple myeloma. Three patients received VA ECMO and the remaining 11 patients received VV ECMO. After being on ECMO for an average of 8.5 days, $50 \%$ of the patients survived the ICU and hospital stay. Their long-term survival was $100 \%$ after a median follow-up of 36 months post discharge [4]. This report was the first of its kind and implied that patients with HMs undergoing ECMO for severe acute respiratory failure may have favorable long-term outcomes.

This accumulated research shows differing conclusions for patients with HMs requiring ECMO for pulmonary support. Whereas studies published earlier suggested that these patients have a mortality rate greater than 50\%, studies from 2014 onward suggest they have a mortality rate less than 50\%. The progression in the literature shows a general transition from seeing poor outcomes for patients with HMs on ECMO to strong outcomes with long-term survival. Although an extensive search was conducted to find more data specifically on patients with HMs on ECMO, it resulted in a limited number of articles. Additional studies are required to draw more reliable conclusions. In addition, three of the four studies mentioned above were retrospective analyses, limiting the ability to control for several potential variables. This calls for systematic prospective studies in the future with larger sample size.

\section{Reports of patients with HMs who were successfully placed on ECMO as a bridge to chemotherapy}

Due to the adverse effects of ECMO, specifically infections, bleeding, and thrombocytopenia, the administration of chemotherapy is not routinely advised in those patients [18]. According to the Extracorporeal Life Support Organization (ELSO), an international nonprofit organization who maintains the worldwide registry of ECMO in active ELSO centers, there are no absolute contraindications to administering chemotherapy while on ECMO [19]. However, ELSO does suggest using extra precautionary measures in patients greater than 65 years of age, and/or those with an absolute neutrophil count of less than $400 / \mathrm{mm}^{3}$ [19-20]. The following cases serve as examples of successful treatment with chemotherapy in patients with HMs requiring ECMO, specifically those with high-grade lymphomas. 
First, Aboud et al. described the case of a 43-year-old woman with newly diagnosed aggressive nonHodgkin's lymphoma who was successfully placed on ECMO as a bridge to curative therapy [21]. Due to massive tumor burden compromising the trachea, she required VV ECMO for imminent hypoxic respiratory failure. While on ECMO, she was treated with lymphoma-specific chemotherapy (Cyclophosphamide, Doxorubicin, Vincristine, Prednisolone, and Rituximab) [21]. The patient was successfully weaned from ECMO six days after admission and was discharged home after a 36-day stay in the ICU. Her subsequent pulmonary function tests were normal, and she achieved complete remission [21].

Next, Worku et al. reported the case of a 41-year-old woman who initially presented with dyspnea due to a 14-cm anterior mediastinal mass encasing the superior vena cava and heart [22]. She was diagnosed with high-grade diffuse large B-cell lymphoma and was started on lymphoma-specific chemotherapy (Dexamethasone, Etoposide, Adriamycin, and Vincristine). Shortly after, she developed cardiogenic shock requiring emergent intubation and intra-aortic balloon pump placement [22]. Her disease-specific prognosis was favorable, considering that diffuse large B-cell lymphoma is typically responsive to appropriate systemic therapy [22]. Hence she was initiated on VA ECMO as a bridge to treatment, and received a five-day course of chemotherapy while on extracorporeal life support. ECMO was discontinued after one cycle of chemotherapy, she was decannulated, and she eventually regained complete autonomous function of her cardiopulmonary circuit. She completed six cycles of chemotherapy and was discharged to a rehabilitation facility after a 144-day hospital stay [22].

Furthermore, Oto et al. described the case of a 40-year-old male with chest pain and dyspnea due to a $12 \mathrm{~cm}$ by $7 \mathrm{~cm}$ mediastinal mass [23]. He underwent tracheal intubation followed immediately by urgent ECMO placement as the tumor completely obstructed the left main bronchus. Fine needle biopsy of the mediastinal tumor was performed, diagnosing precursor T lymphoblastic lymphoma [23]. Chemotherapy with Adriamycin, Vincristine, Cyclophosphamide, and Prednisolone was initiated on hospital day 3 for tumor debulking, after which the mediastinal mass rapidly decreased in size. This was associated with an improvement in oxygenation, and he was weaned from ECMO on hospital day 8 [23]. Subsequent consolidation therapy was administered during his hospital stay, and partial remission of the tumor was confirmed on hospital day 87. The patient proceeded to HSCT with favorable prognosis [23].

Lastly, Allain et al. described the case of a 65 -year-old male who was transferred to the ICU for cardiogenic shock, and soon thereafter was placed on ECMO [24]. A diagnosis of high-grade B cell lymphoma was made and he was started on emergent chemotherapy with Cyclophosphamide, Vindesine, and Solumedrol on day 8 of ECMO support. He was weaned from mechanical support on day 11, and discharged home after a 60-day hospital stay. Six months after discharge, complete remission was confirmed [24]. Without ECMO support, this patient would likely not have recovered from his aggressive B cell lymphoma.

The various examples described above demonstrate that ECMO can serve as a bridge to life-saving, and even curative chemotherapeutic options [21-24]. ECMO can help provide patients the hemodynamic stability they need in order to receive systemic therapy, and thus achieve a long-term disease-free state. In patients with treatment-responsive HMs who are hemodynamically stable on ECMO, and who can tolerate chemotherapy, we suggest that chemotherapy should be considered and administered. The benefits of undergoing ECMO often do outweigh the risks in these patients and can result in mortality benefit, a decision that needs to be assessed on a case-by-case basis. Each patient must be evaluated individually, and comprehensively. Although most of the case reports mentioned above reported positive outcomes, they are still anecdotal studies and are likely subject to bias. There is a need for systematic trials, including prospective studies, which can help provide statistical significance and power to the conclusions made in the case reports we reviewed.

\section{Pharmacokinetics of chemotherapy delivered under ECMO}

The pharmacokinetics of chemotherapy delivered under ECMO has not been sufficiently studied based on the literature review done on this topic. A review article written by Sherwin et al. did establish pharmacokinetic data for anti-infective drugs administered to patients on ECMO [25]. Pharmacokinetics are known to be affected by ECMO in three main ways: 1) direct extraction by the circuit itself, 2) changes in volume of distribution (often increased due to drug extraction and hemodilution in the patient), and 3) altered clearance of the drug (related to renal dysfunction) [25]. Through retrospective data analysis, it was noted that the volume of distribution and clearance of drugs is unchanged in the adult population. Pharmacotherapy dosing recommendations for adults on ECMO are similar to those not requiring ECMO [25]. However, most data on this subject is derived from neonatal studies and case series on adult patients without critically ill controls. The authors have called for further systematic investigation in adult patients to determine better, the proper dosing recommendations in this patient population [25].

Additional data is needed in order to make conclusive recommendations on chemotherapy dosing in patients with HMs who require extracorporeal life support. As ECMO technology is rapidly evolving and being utilized more often in critically ill patients across the world, randomized controlled trials regarding pharmacokinetics of chemotherapy on mechanical support are warranted and desperately needed. These studies are necessary and would contribute to improved treatment of critically ill oncology patients, optimizing outcomes while minimizing toxicity and treatment-related mortality. 


\section{Conclusions}

ECMO is often used in critically ill patients with acute respiratory failure. Differing conclusions regarding its use in patients with concurrent HMs have been reported. Studies published in the late 20th and early 21 st century concluded that patients with HMs on ECMO have poor outcomes. More recent studies published during 2010 to 2019 have suggested a mortality benefit in this patient population, as a result of ECMO support. Reports from 2014 to 2019 have particularly implied that patients with HMs requiring ECMO have an increasingly favorable prognosis, some even exhibiting long-term survival. However, this data consists of small retrospective studies and case reports. Large-scale retrospective and prospective clinical studies should be conducted to establish a stronger conclusion regarding the mortality benefit of ECMO for patients with HMs. Randomized controlled trials studying the pharmacokinetics of chemotherapy in patients on ECMO are also needed to help clinicians reliably dose antineoplastic agents, optimizing outcome and decreasing toxicities. As ECMO becomes more available and continues to gain support in the care of the critically ill, we believe it is important for guidelines to be created to help in the treatment decisions of those patients.

\section{Additional Information \\ Disclosures}

Conflicts of interest: In compliance with the ICMJE uniform disclosure form, all authors declare the following: Payment/services info: All authors have declared that no financial support was received from any organization for the submitted work. Financial relationships: All authors have declared that they have no financial relationships at present or within the previous three years with any organizations that might have an interest in the submitted work. Other relationships: All authors have declared that there are no other relationships or activities that could appear to have influenced the submitted work.

\section{References}

1. Makdisi G, Wang IW: Extra corporeal membrane oxygenation (ECMO) review of a lifesaving technology . J Thorac Dis. 2015, 7:166-176. 10.3978/j.issn.2072-1439.2015.07.17

2. Ratnani I, Tuazon D, Zainab A, Uddin F: The role and impact of extracorporeal membrane oxygenation in critical care. Methodist Debakey Cardiovasc J. 2018, 14:110-119. 10.14797/mdcj-14-2-110

3. Pavlushkov E, Berman M, Valchanov K: Cannulation techniques for extracorporeal life support. Ann Transl Med. 2017, 5:70. 10.21037/atm.2016.11.47

4. Wohlfarth P, Ullrich R, Staudinger T, et al.: Extracorporeal membrane oxygenation in adult patients with hematologic malignancies and severe acute respiratory failure. Crit Care. 2014, 18:20. 10.1186/cc13701

5. Bay JO, Guieze R, Ravinet A, et al.: Major therapeutic advances and new perspectives in onco-hematology (Article in French). Bull Cancer. 2013, 100:587-599. 10.1684/bdc.2013.1751

6. Yeo CD, Kim JW, Kim SC, et al.: Prognostic factors in critically ill patients with hematologic malignancies admitted to the intensive care unit. J Crit Care. 2012, 27:739.e1-739.e6. 10.1016/j.jcrc.2012.07.014

7. Cho S, Cho WC, Lim JY, Kang PJ: Extracorporeal life support in adult patients with hematologic malignancies and acute circulatory and/or respiratory failure. Korean J Thorac Cardiovasc Surg. 2019, 52:2531. 10.5090/kjtcs.2019.52.1.25

8. Prabhu AD, Mos K, Karl TR, Anderson B: Extracorporeal life support in the acute management of tumour lysis syndrome. Interact Cardiovasc Thorac Surg. 2012, 15:568-569. 10.1093/icvts/ivs233

9. Azoulay E, Mokart D, Pène F, et al.: Outcomes of critically ill patients with hematologic malignancies: prospective multicenter data from France and Belgium--a groupe de recherche respiratoire en réanimation onco-hématologique study. J Clin Oncol. 2013, 31:2810. 10.1200/JCO.2012.47.2365

10. Sun HY, Ko WJ, Tsai PR, Sun CC, Chang YY, Lee CW, Chen YC: Infections occurring during extracorporeal membrane oxygenation use in adult patients. J Thorac Cardiovasc Surg. 2010, 140:1125-1132. 10.1016/j.jtcvs.2010.07.017

11. Lee SW, Kim YS, Hong G: Extracorporeal membrane oxygenation as a rescue therapy for acute respiratory failure during chemotherapy in a patient with acute myeloid leukemia. J Thorac Dis. 2017, 9:133-137. $10.21037 /$ jtd.2017.02.23

12. Epner DE, White P, Krasnoff M, Khanduja S, Kimball KT, Knaus WA: Outcome of mechanical ventilation for adults with hematologic malignancy. J Investig Med. 1996, 44:254-260.

13. Gristina GR, Antonelli M, Conti G, et al.: Noninvasive versus invasive ventilation for acute respiratory failure in patients with hematologic malignancies: a 5-year multicenter observational survey. Crit Care Med. 2011, 39:2232-2239. 10.1097/CCM.0b013e3182227a27

14. Ferra C, Marcos P, Misis M, et al.: Outcome and prognostic factors in patients with hematologic malignancies admitted to the intensive care unit: a single-center experience. Int J Hematol. 2007, 85:195202. 10.1532/IJH97.E0625

15. Benoit DD, Vandewoude KH, Decruyenaere JM, Hoste EA, Colardyn FA: Outcome and early prognostic indicators in patients with a hematologic malignancy admitted to the intensive care unit for a lifethreatening complication. Crit Care Med. 2003, 31:104-112. 10.1097/00003246-200301000-00017

16. Gow KW, Lao OB, Leong T, Fortenberry JD: Extracorporeal life support for adults with malignancy and respiratory or cardiac failure: the Extracorporeal Life Support experience. Am J Surg. 2010, 199:669-675. 10.1016/j.amjsurg.2010.01.018

17. Kang HS, Rhee CK, Lee HY, Kim YK, Kwon SS, Kim SC, Lee JW: Clinical outcomes of extracorporeal membrane oxygenation support in patients with hematologic malignancies. Korean J Intern Med. 2015, 30:478-488. 10.3904/kjim.2015.30.4.478

18. Chung JH, Yeo HJ, Cho HM, et al.: Treatment of pulmonary tumor embolism from choriocarcinoma: 


\section{Cureus}

extracorporeal membrane oxygenation as a bridge through chemotherapy. Cancer Res Treat. 2017, 49:279282. $10.4143 / \mathrm{crt} .2016 .125$

19. ELSO guidelines. Extracorporeal life support organization. (2020). Accessed: June 5, 2020: https://www.elso.org/resources/guidelines.aspx.

20. Delnoij TS, Driessen R, Sharma AS, Bouman EA, Strauch U, Roekaerts PM: Venovenous extracorporeal membrane oxygenation in intractable pulmonary insufficiency: practical issues and future directions. Biomed Res Int. 2016, 2016:13. 10.1155/2016/9367464

21. Aboud A, Marx G, Sayer H, Gummert JF: Successful treatment of an aggressive non-Hodgkin's lymphoma associated with acute respiratory insufficiency using extracorporeal membrane oxygenation. Interact Cardiovasc Thorac Surg. 2008, 7:173-174. 10.1510/icvts.2007.159921

22. Worku B, DeBois W, Sobol I, Gulkarov I, Horn EM, Salemi A: Extracorporeal membrane oxygenation as a bridge through chemotherapy in B-cell lymphoma. J Extra Corpor Technol. 2015, 47:52-54.

23. Oto M, Inadomi K, Chosa T, Uneda S, Uekihara S, Yoshida M: Successful use of extracorporeal membrane oxygenation for respiratory failure caused by mediastinal precursor $\mathrm{T}$ lymphoblastic lymphoma. Case Rep Med. 2014, 2014:4. 10.1155/2014/804917

24. Allain G, Hajj-Chahine J, Lacroix C, Jayle C: Primary cardiac lymphoma complicated by cardiogenic shock: successful treatment with chemotherapy delivered under extracorporeal membrane oxygenation support. Eur J Cardiothorac Surg. 2015, 48:968-970. 10.1093/ejcts/ezv031

25. Sherwin J, Heath T, Watt K: Pharmacokinetics and dosing of anti-infective drugs in patients on extracorporeal membrane oxygenation: a review of the current literature. Clin Ther. 2016, 38:1976-1994. 10.1016/j.clinthera.2016.07.169 